\title{
On hyperbolicity of the dynamic equations for plastic fluid-saturated solids
}

Received: 9 February 2021 / Accepted: 24 April 2021 / Published online: 12 May 2021

(C) The Author(s) 2021

\begin{abstract}
The paper deals with the analysis of hyperbolicity of the dynamic equations for plastic solids, including one-phase solids and porous fluid-saturated solids with zero and nonzero permeability. Hyperbolicity defined as diagonalizability of the matrix of the system is necessary for the boundary value problems to be well posed. The difference between the system of equations for a plastic solid and the system for an elastic solid is that the former contains additional evolution equations for the dependent variables involved in the plasticity model. It is shown that the two systems agree with each other from the viewpoint of hyperbolicity: they are either both hyperbolic or both non-hyperbolic. Another issue addressed in the paper is the relation between hyperbolicity and the properties of the acoustic tensor (matrix). It remained unproved whether the condition for the eigenvalues of the acoustic matrix to be real and positive is not only necessary but also sufficient for hyperbolicity. It is proved in the paper that the equations are hyperbolic if and only if the eigenvalues of the acoustic matrix are real and positive with a complete set of eigenvectors. The analysis of the whole system of equations for a plastic solid can thus be reduced to the analysis of the acoustic matrix. The results are not restricted to a particular plasticity model but applicable to a wide class of models.
\end{abstract}

Keywords Hyperbolicity · Plasticity · Fluid-saturated solid · Acoustic tensor

\section{Introduction}

An important question arising in connection with any boundary value problem is whether the problem is well posed. Well-posedness is defined as the existence of a unique solution which depends continuously on the initial and boundary data. Well-posedness of dynamic problems for solids with rate-independent constitutive behaviour requires the system of the governing equations to be hyperbolic. This concerns both one-phase solids and porous fluid-saturated solids (with exceptions mentioned in Sect. 2.4). Whereas well-posedness may be difficult to prove even for relatively simple problems, hyperbolicity as a necessary condition for well-posedness can be verified numerically in many cases without considerable difficulties.

In relation to systems of first-order partial differential equations considered in this paper, the requirement for a system to be hyperbolic imposes conditions on the eigenvalues and eigenvectors of the matrix of the system. The eigenvalues (the characteristic speeds) must be real with a complete set of eigenvectors (the exact definition is given in Sect. 2.1). The equations of motion for the velocities and stresses as unknown functions and the stress-strain relations in rate form constitute a closed system of first-order equations from which the characteristic speeds can be found. The stiffness tensor in the stress-strain relations can be either constant, if the solid is elastic, or obtained from the plasticity model employed. A possible loss of hyperbolicity due to complex characteristic speeds and the resulting ill-posedness of the problem may have different interpretations. Purely

V. A. Osinov $(\bowtie)$

Institute of Soil Mechanics and Rock Mechanics, Karlsruhe Institute of Technology, 76128 Karlsruhe, Germany

E-mail: vladimir.osinov@kit.edu 
imaginary characteristic speeds may reflect the real behaviour of the solid (the localization of deformation) and thus be physically justified [1,2]. In contrast, complex conjugate speeds leading to 'flutter instability' [2] should most probably be regarded as a consequence of incorrect constitutive modelling, as there has been no physical justification for this case so far.

The present paper addresses hyperbolicity of three systems of dynamic equations: for one-phase solids, for fluid-saturated solids with zero permeability and for fluid-saturated solids with nonzero permeability. Previous studies on hyperbolicity of the dynamic equations for plastic one-phase and fluid-saturated solids were restricted to the analysis of the characteristic speeds without considering the eigenvectors of the matrix of the system [3-9]. Owing to this restriction, two issues concerning hyperbolicity remained unresolved. They are considered in the present paper. The first one is the influence of the plastic behaviour. Finding the characteristic speeds for a plastic solid actually reduces to the analysis of an elastic solid whose stiffness tensor is the same as the current stiffness tensor of the plasticity model, taking into account loading and unloading when dealing with elasto-plasticity. However, in the case of a plastic solid the equations of motion and the stress-strain relations are only part of the whole system. Plasticity models typically involve additional dependent variables such as plastic strains and hardening parameters with their own evolution equations. Any additional equations increase the size of the eigenvectors and the number or multiplicity of the eigenvalues. It was not analysed rigorously whether hyperbolicity of the reduced system that includes only the equations of motion and the stress-strain relations is always in agreement with hyperbolicity of the extended system that also includes the additional evolution equations.

A proposition proved in Sect. 3 establishes the equivalence between the reduced and extended systems from the viewpoint of hyperbolicity. It is shown that the systems are either both hyperbolic or both non-hyperbolic. The analysis is not restricted to a particular plasticity model. The additional dependent variables and their evolution equations responsible for the plastic behaviour are introduced on rather general assumptions which conform with the majority of plasticity models, thus enabling a wide range of applications.

The second issue addressed in the paper is the connection between hyperbolicity and the properties of the acoustic tensor (one-phase solids) or the 'acoustic matrix' (fluid-saturated solids). It is known that the characteristic speeds are real if the eigenvalues of the acoustic matrix are real and positive. The eigenvalues of the acoustic matrix allow us to find the characteristic speeds, but it is not evident that the real and positive eigenvalues of the acoustic matrix guarantee the existence of a complete set of linearly independent eigenvectors of the matrix of the system. This latter matrix is larger in size than the acoustic matrix and has additional zero eigenvalues. A proposition proved in Sect. 4 establishes the required connection between the acoustic matrix and the matrix of the system.

The two propositions applied together show that the properties of the acoustic matrix fully determine hyperbolicity of the whole system of equations for a plastic solid. The equations are hyperbolic if and only if the eigenvalues of the acoustic matrix are real and positive with a complete set of eigenvectors.

\section{Dynamic equations}

\subsection{Definition of hyperbolicity}

The equations studied in this paper are systems of first-order partial differential equations

$$
\frac{\partial u_{i}}{\partial t}+\sum_{k=1}^{3} M_{i j}^{(k)} \frac{\partial u_{j}}{\partial x_{k}}=F_{i}, \quad i=1, \ldots, N,
$$

where $u_{1}, \ldots, u_{N}$ are functions of Cartesian coordinates $x_{1}, x_{2}, x_{3}$ and time $t$ and $M_{i j}^{(k)}$ are the components of real $N \times N$ matrices $M^{(k)}, k=1,2,3$. The summation convention for repeated indices is used throughout the paper. The matrices $M^{(k)}$ and the right-hand sides $F_{i}$ may be functions of the dependent and independent variables.

Definition (hyperbolicity) System (1) is called hyperbolic if for any real $n_{1}, n_{2}, n_{3}$ the matrix $M=$ $\sum_{k=1}^{3} n_{k} M^{(k)}$ is diagonalizable by a real matrix ([10], Sect. 7.3.1). 
Equivalently, the system is called hyperbolic if for any real $n_{1}, n_{2}, n_{3}$ the matrix $M$ has $N$ linearly independent real eigenvectors. The verification of hyperbolicity amounts to the analysis of the eigenvalue problem

$$
M_{i j} u_{j}^{0}=c u_{i}^{0}, \quad i=1, \ldots, N,
$$

where $M_{i j}$ are the components of the matrix $M$ and $u_{i}^{0}$ are the components of an eigenvector associated with an eigenvalue $c$. Without loss of generality, the factors $n_{1}, n_{2}, n_{3}$ in the definition of the matrix $M$ will be taken to be the components of a unit vector $\mathbf{n}$. In this case, the eigenvalues $c$ are referred to as the characteristic speeds since they coincide with the speeds of plane waves that propagate in the direction $\mathbf{n}$ and are solutions to a homogeneous system (1) with constant coefficients.

Note that hyperbolicity is sometimes defined in a weaker sense with the only requirement that the eigenvalues of the matrix $M$ be real, without imposing the condition of the existence of a complete set of eigenvectors. (Systems whose matrix has real eigenvalues and fewer than $N$ linearly independent eigenvectors are classified by some authors as parabolic, see e.g. [11], Sect. 3.3.) Note also that the conditions of strict hyperbolicity, when the eigenvalues of the matrix $M$ must be not only real but also all different, are too strong and a priori not satisfied by the equations studied here. Even in the simplest case of an isotropic solid in three-dimensional problems, the matrix $M$ has a double eigenvalue that corresponds to transverse waves. Moreover, there always exists a multiple eigenvalue equal to zero.

\subsection{One-phase solids}

The dynamic equations written in a Cartesian coordinate system $\left(x_{1}, x_{2}, x_{3}\right)$ for the velocities and stresses as dependent variables are first-order equations of the form (1). In the following, the material time derivatives in the equations will be replaced with the partial time derivatives neglecting the convective terms. The system of equations for a one-phase solid includes the equations of motion,

$$
\frac{\partial \sigma_{j i}}{\partial x_{j}}=\varrho \frac{\partial v_{i}}{\partial t},
$$

and the constitutive equations in rate form,

$$
\frac{\partial \sigma_{j i}}{\partial t}=C_{j i k l} \frac{\partial v_{k}}{\partial x_{l}},
$$

where $v_{i}, \sigma_{j i}$ and $C_{j i k l}$ are, respectively, the Cartesian components of the velocity vector, the stress tensor and the stiffness tensor, and $\varrho$ is the density. For a porous solid, $\varrho=(1-n) \varrho_{s}$, where $n$ is the porosity and $\varrho_{s}$ is the density of the solid phase. System (3), (4) with constant coefficients for the functions $v_{i}, \sigma_{j i}$ (where $\sigma_{j i}=\sigma_{i j}$ ) describes the dynamic deformation of a linearly elastic solid.

The eigenvalue problem (2) obtained from system (3), (4) is

$$
\begin{aligned}
& -\frac{1}{\varrho} n_{j} \sigma_{j i}^{0}=c v_{i}^{0}, \\
& -C_{j i k l} n_{l} v_{k}^{0}=c \sigma_{j i}^{0},
\end{aligned}
$$

where $v_{i}^{0}, \sigma_{j i}^{0}$ are the components of an eigenvector. If the components are ordered as

$$
(\underbrace{v_{1}^{0}, v_{2}^{0}, v_{3}^{0}}_{N_{1}}, \underbrace{\sigma_{11}^{0}, \sigma_{12}^{0}, \sigma_{13}^{0}, \sigma_{22}^{0}, \sigma_{33}^{0}, \sigma_{23}^{0}}_{N_{2}})^{T},
$$

then the matrix in (5), (6), denoted by $R$, has the structure

$$
R_{N \times N}=\left(\begin{array}{cc}
0_{N_{1} \times N_{1}} & Y_{N_{1} \times N_{2}} \\
X_{N_{2} \times N_{1}} & 0_{N_{2} \times N_{2}}
\end{array}\right), \quad N=N_{1}+N_{2}, \quad N_{1} \leq N_{2},
$$

where $0_{N_{1} \times N_{1}}, 0_{N_{2} \times N_{2}}$ are zero matrices and $X_{N_{2} \times N_{1}}, Y_{N_{1} \times N_{2}}$ are nonzero matrices. 
The matrix $R$ determined by (5), (6) always has a zero eigenvalue with at least three linearly independent eigenvectors. Let $\mathbf{n}$ be given and $s_{i}, q_{i}, i=1,2,3$, be the components of two unit vectors $\mathbf{s}, \mathbf{q}$ orthogonal to each other and to the vector $\mathbf{n}$. It can be verified directly that the three vectors

$$
\begin{aligned}
v_{i}^{0}=0, & \sigma_{j i}^{0}=s_{j} s_{i}, \\
v_{i}^{0}=0, & \sigma_{j i}^{0}=q_{j} q_{i}, \\
v_{i}^{0}=0, & \sigma_{j i}^{0}=s_{j} q_{i}+s_{i} q_{j}
\end{aligned}
$$

are solutions to (5), (6) with $c=0$. To see that the three eigenvectors (9-11) are linearly independent, extend the 9 -component vectors (7) to 12-component ones by adding the components $\sigma_{21}^{0}, \sigma_{31}^{0}, \sigma_{32}^{0}$. If three 12-component vectors obtained in such a way are linearly independent, then the corresponding 9-component vectors are linearly independent as well. It is readily seen that the three eigenvectors (9-11) treated as 12component vectors are mutually orthogonal and therefore linearly independent.

System (3), (4) with constant coefficients which describes an elastic solid will be referred to as the reduced system and the corresponding matrix $R$ of the eigenvalue problem (5), (6) as the reduced matrix.

Now assume that the solid undergoes plastic deformation. The incremental constitutive response in plasticity models is typically piecewise linear with two or more constitutive cones with different stiffness tensors. Hyperbolicity should be verified separately for each constitutive cone. Equations (3), (4) remain valid for plastic solids but are only part of the governing equations. In order to extend the analysis to plastic solids without considering particular plasticity models, assume that

(i) the velocities and stresses are supplemented with additional dependent variables $\psi_{1}, \ldots, \psi_{L}$, which may be scalars or components of tensors,

(ii) the evolution of the additional dependent variables is determined by the deformation of the solid and is governed by equations of the form

$$
\frac{\partial \psi_{i}}{\partial t}+\sum_{k=1}^{3} S_{i j}^{(k)} \frac{\partial v_{j}}{\partial x_{k}}=0, \quad i=1, \ldots, L
$$

where $S_{i j}^{(k)}$ are the components of $L \times 3$ matrices $S^{(k)}, k=1,2,3$,

(iii) the coefficients in the equations may be functions of the dependent variables including $\psi_{1}, \ldots, \psi_{L}$.

The reduced system supplemented with the new functions and equations according to the assumptions (i-iii) will be referred to as the extended system and its matrix as the extended matrix. The assumptions (iiii) cover a wide class of plasticity models with rate-independent constitutive behaviour. In elasto-plasticity, the dependent variables $\psi_{i}$ may represent plastic strains and hardening parameters, e.g. plastic work or back stresses. The present analysis is also valid for rate-dependent visco-plasticity models of the Perzyna type [12] which contain additional functions of the dependent variables in equations (4), (12). Such terms do not involve derivatives of the dependent variables and therefore have no effect on hyperbolicity.

The eigenvalue problem (5), (6) of the reduced system is supplemented with the new equations

$$
\sum_{k=1}^{3} n_{k} S_{i j}^{(k)} v_{j}^{0}=c \psi_{i}^{0}, \quad i=1, \ldots, L,
$$

where $\psi_{i}^{0}$ are the additional components of the eigenvectors. If the components are ordered as

$$
(\underbrace{v_{1}^{0}, v_{2}^{0}, v_{3}^{0}}_{N_{1}}, \underbrace{\sigma_{11}^{0}, \sigma_{12}^{0}, \sigma_{13}^{0}, \sigma_{22}^{0}, \sigma_{33}^{0}, \sigma_{23}^{0}}_{N_{2}}, \psi_{1}^{0}, \ldots, \psi_{L}^{0})^{T},
$$

then the extended matrix, denoted by $E$, has the structure

$$
E_{(N+L) \times(N+L)}=\left(\begin{array}{ccc}
0_{N_{1} \times N_{1}} & Y_{N_{1} \times N_{2}} & 0_{N_{1} \times L} \\
X_{N_{2} \times N_{1}} & 0_{N_{2} \times N_{2}} & 0_{N_{2} \times L} \\
Z_{L \times N_{1}} & 0_{L \times N_{2}} & 0_{L \times L}
\end{array}\right), \quad N=N_{1}+N_{2}, \quad N_{1} \leq N_{2},
$$

where $Z_{L \times N_{1}}$ is a nonzero matrix. For equations (12), (13), $Z_{L \times 3}=\sum_{k=1}^{3} n_{k} S^{(k)}$. 


\subsection{Fluid-saturated solids with zero permeability}

Considering porous fluid-saturated solids, we assume that the solid skeleton as a whole is much more compressible than the solid phase itself. This assumption is usually formulated as incompressibility of the solid phase and is justified, for instance, for soils. The constitutive equations (4) for a dry solid skeleton remain valid for the same but saturated skeleton. They relate the rate of the skeleton deformation to the rate of the effective stresses $\sigma_{j i}$. For a porous medium with an incompressible solid phase, the effective stresses are

$$
\sigma_{j i}=\sigma_{j i}^{\text {total }}+p_{f} \delta_{j i}
$$

where $\sigma_{j i}^{\text {total }}$ are the total stresses, $p_{f}$ is the pore pressure (positive for compression) and $\delta_{j i}$ is the Kronecker delta.

For zero skeleton permeability (locally undrained conditions), the velocity field is common to both the skeleton and the fluid. The equations of motion are written for the total stress:

$$
\frac{\partial \sigma_{j i}^{\text {total }}}{\partial x_{j}}=\varrho \frac{\partial v_{i}}{\partial t}
$$

where

$$
\varrho=(1-n) \varrho_{s}+n \varrho_{f}
$$

is the density of the medium, $\varrho_{s}$ and $\varrho_{f}$ are the densities of the solid and fluid phases and $n$ is the skeleton porosity. Changes in the pore pressure are determined by the evolution equation

$$
\frac{\partial p_{f}}{\partial t}=-\frac{K_{f}}{n} \frac{\partial v_{k}}{\partial x_{k}}
$$

where $K_{f}$ is the pore fluid bulk modulus [13]. Equations (4), (16), (19) yield the constitutive relations for the total stress

$$
\frac{\partial \sigma_{j i}^{t o t a l}}{\partial t}=\left(C_{j i k l}+\frac{K_{f}}{n} \delta_{j i} \delta_{k l}\right) \frac{\partial v_{k}}{\partial x_{l}} .
$$

Equations (17), (20) with constant coefficients for the functions $v_{i}, \sigma_{j i}^{\text {total }}$ describe the dynamic deformation of a saturated solid with an elastic skeleton. The governing equations and the corresponding eigenvalue problem are similar to those for a one-phase solid. Equations (5) of the eigenvalue problem remain unchanged, and equations (6) become

$$
-\left(C_{j i k l}+\frac{K_{f}}{n} \delta_{j i} \delta_{k l}\right) n_{l} v_{k}^{0}=c \sigma_{j i}^{0}
$$

where $\sigma_{j i}^{0}$ now stands for the total stress components. If the dependent variables are ordered as in (7), the matrix of the system has the structure (8). The matrix has the same three linearly independent eigenvectors (9-11) associated with the zero eigenvalue.

For the plastic behaviour of the skeleton, we make the same assumptions (i-iii) of Sect. 2.2 to obtain an extended system and an extended matrix (15). The stiffness of a plastic skeleton usually depends, among other quantities, on the effective stresses, which are not available in Eqs. (17), (20). The simplest way to gain the effective stresses is to introduce the pore pressure $p_{f}$ as a new dependent variable $\psi_{i}$ with the evolution Eq. (19) which conforms with (12). The pore pressure as a separate dependent variable may also be needed if, for instance, the pore fluid consists of water and a small amount of free (undissolved) gas. Such a medium may still be modelled as a two-phase medium if the gaseous phase is not continuous but is in the form of separate inclusions (bubbles) in the liquid phase. The free gas makes the pore fluid bulk modulus $K_{f}$ strongly pore pressure dependent [14]. 


\subsection{Fluid-saturated solids with nonzero permeability}

In a fluid-saturated solid with nonzero permeability (locally drained conditions), the solid skeleton and the pore fluid have in general different velocities. The velocity components for the solid and fluid phases will be denoted, respectively, by $v_{s i}$ and $v_{f i}$, where the first subscript stands for the phase and the second one indicates the Cartesian component. The effective stresses $\sigma_{j i}$ are defined by (16) on the assumption that the solid phase is incompressible. The equations of motion are written separately for the solid and fluid phases [13]:

$$
\begin{aligned}
& \frac{\partial \sigma_{j i}}{\partial x_{j}}-(1-n) \frac{\partial p_{f}}{\partial x_{i}}+\frac{\varrho_{f} g n^{2}}{k}\left(v_{f i}-v_{s i}\right)=(1-n) \varrho_{s} \frac{\partial v_{s i}}{\partial t}, \\
& -n \frac{\partial p_{f}}{\partial x_{i}}-\frac{\varrho_{f} g n^{2}}{k}\left(v_{f i}-v_{s i}\right)=n \varrho_{f} \frac{\partial v_{f i}}{\partial t}
\end{aligned}
$$

where $k$ is the skeleton permeability $(\mathrm{m} / \mathrm{s})$ and $g$ is the acceleration due to gravity. The constitutive relations (4) for a dry skeleton remain valid for the effective stresses but are now written in terms of the skeleton velocity:

$$
\frac{\partial \sigma_{j i}}{\partial t}=C_{j i k l} \frac{\partial v_{s k}}{\partial x_{l}} .
$$

The evolution equation for the pore pressure reads [13]

$$
\frac{\partial p_{f}}{\partial t}=-\frac{K_{f}}{n}\left[(1-n) \frac{\partial v_{s k}}{\partial x_{k}}+n \frac{\partial v_{f k}}{\partial x_{k}}+\left(v_{f k}-v_{s k}\right) \frac{\partial n}{\partial x_{k}}\right] .
$$

If the skeleton stiffness tensor and the pore fluid bulk modulus do not change with the deformation and temporal changes in the porosity are neglected, then Eqs. (22-25) for the unknown functions $v_{s i}, v_{f i}, \sigma_{j i}, p_{f}$ describe the dynamic deformation of a saturated solid with an elastic skeleton.

In connection with the dynamic equations for fluid-saturated solids, note that there are two cases to which the subsequent analysis does not apply: the case of incompressible constituents and the so-called $u$ - $p$-approximation. If both the solid and fluid phases are assumed to be incompressible, then Eq. (25) with $K_{f} \rightarrow \infty$ becomes the incompressibility condition for the two velocity fields. This condition does not contain any time derivative and is not in the form (1). In the $u$-p-approximation used for sufficiently slow processes such as earthquake-induced deformation [13], the solid and fluid phases are assumed to have the same acceleration, so that the fluid acceleration $\partial v_{f i} / \partial t$ in the equation of motion (23) for the fluid phase is replaced with the acceleration of the solid phase $\partial v_{s i} / \partial t$. As a consequence, there are two equations, (22) and (23), with the acceleration of the solid and no equation with the acceleration of the fluid, and the system is again not in the form (1).

The eigenvalue problem obtained from Eqs. (22-25) is

$$
\begin{aligned}
& \frac{1}{(1-n) \varrho_{s}}\left[-n_{j} \sigma_{j i}^{0}+(1-n) n_{i} p_{f}^{0}\right]=c v_{s i}^{0}, \\
& \frac{1}{\varrho_{f}} n_{i} p_{f}^{0}=c v_{f i}^{0} \\
& -C_{j i k l} n_{l} v_{s k}^{0}=c \sigma_{j i}^{0} \\
& K_{f}\left(\frac{1-n}{n}\right) n_{k} v_{s k}^{0}+K_{f} n_{k} v_{f k}^{0}=c p_{f}^{0}
\end{aligned}
$$

where $v_{s i}^{0}, v_{f i}^{0}, \sigma_{j i}^{0}, p_{f}^{0}$ are the components of an eigenvector. If the components are ordered as

$$
(\underbrace{v_{s 1}^{0}, v_{s 2}^{0}, v_{s 3}^{0}, v_{f 1}^{0}, v_{f 2}^{0}, v_{f 3}^{0}}_{N_{1}}, \underbrace{\sigma_{11}^{0}, \sigma_{12}^{0}, \sigma_{13}^{0}, \sigma_{22}^{0}, \sigma_{33}^{0}, \sigma_{23}^{0}, p_{f}^{0}}_{N_{2}})^{T},
$$

then the matrix of the system has the structure (8). 
The eigenvalue problem (26-29) yields a zero eigenvalue with at least 5 linearly independent eigenvectors. Let $\mathbf{s , q}$ be two unit vectors orthogonal to each other and to the vector $\mathbf{n}$. It can be verified directly that the following 5 vectors are solutions to (26-29) with $c=0$ :

$$
\begin{aligned}
& v_{s i}^{0}=0, \quad v_{f i}^{0}=0, \quad \sigma_{j i}^{0}=s_{j} s_{i}, \quad p_{f}^{0}=0, \\
& v_{s i}^{0}=0, \quad v_{f i}^{0}=0, \quad \sigma_{j i}^{0}=q_{j} q_{i}, \quad p_{f}^{0}=0, \\
& v_{s i}^{0}=0, \quad v_{f i}^{0}=0, \quad \sigma_{j i}^{0}=s_{j} q_{i}+s_{i} q_{j}, \quad p_{f}^{0}=0, \\
& v_{s i}^{0}=0, \quad v_{f i}^{0}=s_{i}, \quad \sigma_{j i}^{0}=0, \quad p_{f}^{0}=0, \\
& v_{s i}^{0}=0, \quad v_{f i}^{0}=q_{i}, \quad \sigma_{j i}^{0}=0, \quad p_{f}^{0}=0 .
\end{aligned}
$$

The proof that the eigenvectors (31-35) are linearly independent is in essence the same as for the eigenvectors (9-11).

For plastic deformation, we may slightly generalize the assumption (ii) of Sect. 2.2 and assume that the evolution of the additional functions $\psi_{i}$ may depend not only on the strain rate of the skeleton but also on the volumetric strain rate of the pore fluid. Equations (12) take the form

$$
\frac{\partial \psi_{i}}{\partial t}+\sum_{k=1}^{3} S_{i j}^{(k)} \frac{\partial v_{s j}}{\partial x_{k}}+\beta_{i} \frac{\partial v_{f j}}{\partial x_{j}}=0, \quad i=1, \ldots, L,
$$

with additional coefficients $\beta_{i}$. The eigenvalue problem (26-29) is supplemented with the equations

$$
\sum_{k=1}^{3} n_{k} S_{i j}^{(k)} v_{s j}^{0}+\beta_{i} n_{j} v_{f j}^{0}=c \psi_{i}^{0}, \quad i=1, \ldots, L .
$$

If the vector components in the eigenvalue problem are ordered as

$$
(\underbrace{v_{s 1}^{0}, v_{s 2}^{0}, v_{s 3}^{0}, v_{f 1}^{0}, v_{f 2}^{0}, v_{f 3}^{0}}_{N_{1}}, \underbrace{\sigma_{11}^{0}, \sigma_{12}^{0}, \sigma_{13}^{0}, \sigma_{22}^{0}, \sigma_{33}^{0}, \sigma_{23}^{0}, p_{f}^{0}}_{N_{2}}, \psi_{1}^{0}, \ldots, \psi_{L}^{0})^{T},
$$

then the extended matrix $E$ has the structure (15) with

$$
Z_{L \times 6}=\left(\begin{array}{cc}
S_{L \times 3} & B_{L \times 3}
\end{array}\right),
$$

where the matrices $S$ and $B$ have the components

$$
S_{i j}=\sum_{k=1}^{3} n_{k} S_{i j}^{(k)}, \quad B_{i j}=\beta_{i} n_{j}
$$

Special remarks should be made about the porosity $n$ in the case of nonzero permeability considered in this section. The proof of a proposition in Sect. 3 will be based on the fact that the matrices $R$ and $E$ have the special structures (8), (15). The last term in the evolution equation (25) for the pore pressure contains the porosity gradient. If the porosity is treated as a constant field, as was assumed in the elastic case, the term with the porosity gradient in (25) belongs to the right-hand side of the system (in the notations of (1)) and has no effect on the matrices $R$ and $E$. If the porosity appears as a dependent variable $\psi_{i}$ in a plasticity model, its changes are determined, for an incompressible solid phase, by the evolution equation

$$
\frac{\partial n}{\partial t}=(1-n) \frac{\partial v_{s j}}{\partial x_{j}}
$$

Although Eq. (41) conforms with (36), the consequence of including the porosity in the set of dependent variables is that the term with the porosity gradient in (25) moves to the left-hand side of the system and breaks the structure of the matrix $E$ shown in (15). Such cases are not considered in this paper. Neglecting the last term in (25) with the porosity gradient makes the results applicable to plasticity models with the variable porosity described by Eq. (41).

The equations of Sects. 2.2-2.4 are summarized in Table 1. 
Table 1 Equations of the reduced and extended systems (elastic and plastic solids, respectively)

\begin{tabular}{|c|c|c|c|c|}
\hline & & Governing equations & Eigenvalue problem & Eigenvectors associated with $c=0$ \\
\hline I & $\begin{array}{l}\text { One-phase solid } \\
\text { (a) elastic } \\
\text { (b) plastic }\end{array}$ & $\begin{array}{l}(3),(4) \\
(3),(4),(12)\end{array}$ & $\begin{array}{l}(5),(6) \\
(5),(6),(13)\end{array}$ & $\begin{array}{l}(9)-(11) \\
(57),(61),(62)\end{array}$ \\
\hline II & $\begin{array}{l}\text { Saturated solid with zero permeability } \\
\text { (a) elastic } \\
\text { (b) plastic }\end{array}$ & $\begin{array}{l}(17),(20) \\
(17),(20),(12)\end{array}$ & $\begin{array}{l}(5),(6) \\
(5),(6),(13)\end{array}$ & $\begin{array}{l}(9)-(11) \\
(57),(61),(62)\end{array}$ \\
\hline III & $\begin{array}{l}\text { Saturated solid with nonzero permeability } \\
\text { (a) elastic } \\
\text { (b) plastic }\end{array}$ & $\begin{array}{l}(22)-(25) \\
(22)-(25),(36)\end{array}$ & $\begin{array}{l}(26)-(29) \\
(26)-(29),(37)\end{array}$ & $\begin{array}{l}(31)-(35) \\
(57),(61),(62)\end{array}$ \\
\hline
\end{tabular}

\section{Hyperbolicity of the reduced and extended systems}

For the proof of hyperbolicity, we first need a lemma. In the following, $\operatorname{dim} \operatorname{ker}(A)$ denotes the dimension of the nullspace (the kernel) of a matrix $A$, and alg mult $A_{A}(\lambda)$ denotes the algebraic multiplicity of the eigenvalue $\lambda$ of a matrix $A$.

Lemma If a square matrix $R$ of the form (8) is such that

$$
\operatorname{dim} \operatorname{ker}(R)=\operatorname{alg} \operatorname{mult}_{R}(0),
$$

then

$$
\operatorname{dim} \operatorname{ker}(R)=2 \operatorname{dim} \operatorname{ker}(Y)-N_{2}+N_{1}
$$

Proof For any square matrix $R$,

$$
\operatorname{alg} \operatorname{mult}_{R}(0)=\operatorname{alg}_{\operatorname{mult}_{R^{2}}}(0)
$$

For a matrix $R$ of the form (8),

$$
\begin{aligned}
R_{N \times N}^{2} & =\left(\begin{array}{cc}
Y X_{N_{1} \times N_{1}} & 0_{N_{1} \times N_{2}} \\
0_{N_{2} \times N_{1}} & X Y_{N_{2} \times N_{2}}
\end{array}\right), \\
\operatorname{alg~mult~}_{R^{2}}(0) & =\operatorname{alg} \operatorname{mult}_{Y X}(0)+\operatorname{alg} \operatorname{mult}_{X Y}(0) .
\end{aligned}
$$

Due to the special structure (8) of the matrix $R$,

$$
\operatorname{dim} \operatorname{ker}(R)=\operatorname{dim} \operatorname{ker}(X)+\operatorname{dim} \operatorname{ker}(Y) .
$$

Equations (47), (42), (44), (46) used successively lead to

$$
\operatorname{dim} \operatorname{ker}(X)+\operatorname{dim} \operatorname{ker}(Y)=\operatorname{alg} \operatorname{mult}_{Y X}(0)+\operatorname{alg} \operatorname{mult}_{X Y}(0) .
$$

As follows from the general inequalities

$$
\begin{aligned}
& \operatorname{dim} \operatorname{ker}(X) \leq \operatorname{dim} \operatorname{ker}(Y X) \leq \operatorname{alg}^{\operatorname{mult}_{Y X}(0),} \\
& \operatorname{dim} \operatorname{ker}(Y) \leq \operatorname{dim} \operatorname{ker}(X Y) \leq \operatorname{alg} \operatorname{mult}_{X Y}(0),
\end{aligned}
$$

equality (48) is possible only if

$$
\begin{aligned}
& \operatorname{dim} \operatorname{ker}(X)=\operatorname{alg} \operatorname{mult}_{Y X}(0), \\
& \operatorname{dim} \operatorname{ker}(Y)=\operatorname{alg} \text { mult }_{X Y}(0) .
\end{aligned}
$$

Taking into account the relation

$$
\operatorname{alg} \operatorname{mult}_{X Y}(0)=\operatorname{alg} \operatorname{mult}_{Y X}(0)+N_{2}-N_{1}
$$

([15], p. 53, [16], Exercise 6.2.16), we obtain

$$
\operatorname{dim} \operatorname{ker}(Y)=\operatorname{dim} \operatorname{ker}(X)+N_{2}-N_{1} .
$$

Equality (43) follows from (47) and (54). 
The next objective is to show the equivalence between the reduced and extended systems with respect to hyperbolicity. It is seen from (8), (15) that for a given $\mathbf{n}$ the reduced and extended matrices have the same eigenvalues because

$$
\operatorname{det}\left(E-c I_{E}\right)=(-c)^{L} \operatorname{det}\left(R-c I_{R}\right),
$$

where $I_{E}, I_{R}$ are the $(L+N) \times(L+N)$ and $N \times N$ unit matrices, respectively. This property would suffice for the equivalence of the two systems if hyperbolicity were defined in the weak sense mentioned in Sect. 2.1. For the definition adopted here, the identity of the spectra that follows from (55) does not suffice as it does not provide the required information about the eigenvectors of the matrices. The equivalence is established by the following proposition valid for the reduced and extended systems I-III listed in Table 1. See also the remarks about the porosity at the end of Sect. 2.4.

Proposition 1 A reduced system and the corresponding extended system are either both hyperbolic or both non-hyperbolic.

Proof For a given $\mathbf{n}$, let

$$
\left(u_{1}^{0}, \ldots, u_{N}^{0}\right)^{T}
$$

denote an eigenvector of the reduced matrix. Suppose that the reduced matrix has a complete set of linearly independent eigenvectors. If (56) is an eigenvector associated with $c \neq 0$, then $c$ is also an eigenvalue of the extended matrix with the eigenvector

$$
\left(u_{1}^{0}, \ldots, u_{N}^{0}, \psi_{1}^{0}, \ldots, \psi_{L}^{0}\right)^{T},
$$

where $u_{1}^{0}, \ldots, u_{N}^{0}$ are the same as in (56) and $\psi_{i}^{0}$ are determined by (13) or (37). The eigenvectors of the extended matrix obtained in this way from a set of linearly independent eigenvectors of the reduced matrix are linearly independent as well. The eigenvectors of the reduced matrices associated with $c=0$ are determined by the equations listed in the last column of Table 1. A key question is whether, for each reduced matrix, the eigenvectors determined by those equations span the entire nullspace of the matrix or only a subspace of the nullspace. The question can be answered with the help of the lemma proved above. For the use of the lemma, we need to know the ranks of the matrices $Y$ contained in $R$. For systems I,II, the matrix $Y$ determined by (5) is

$$
Y_{3 \times 6}=-\frac{1}{\varrho}\left(\begin{array}{cccccc}
n_{1} & n_{2} & n_{3} & 0 & 0 & 0 \\
0 & n_{1} & 0 & n_{2} & 0 & n_{3} \\
0 & 0 & n_{1} & 0 & n_{3} & n_{2}
\end{array}\right) .
$$

The rank of this matrix is 3 for any $\mathbf{n}$. For system III, the matrix $Y$ determined by (26), (27) is

$$
Y_{6 \times 7}=-\frac{1}{(1-n) \varrho_{s}}\left(\begin{array}{ccccccc}
n_{1} & n_{2} & n_{3} & 0 & 0 & 0 & -(1-n) n_{1} \\
0 & n_{1} & 0 & n_{2} & 0 & n_{3} & -(1-n) n_{2} \\
0 & 0 & n_{1} & 0 & n_{3} & n_{2} & -(1-n) n_{3} \\
0 & 0 & 0 & 0 & 0 & 0 & -(1-n) \varrho_{s} n_{1} / \varrho_{f} \\
0 & 0 & 0 & 0 & 0 & 0 & -(1-n) \varrho_{s} n_{2} / \varrho_{f} \\
0 & 0 & 0 & 0 & 0 & 0 & -(1-n) \varrho_{s} n_{3} / \varrho_{f}
\end{array}\right) .
$$

The rank of this matrix is 4 for any $\mathbf{n}$. To show this, assume without loss of generality that $n_{1} \neq 0$ and make the 5th and 6th rows zero with the help of the 4th row. Having found the ranks of the two matrices $Y$, we obtain, using the rank-nullity theorem,

$$
\operatorname{dim} \operatorname{ker}(Y)=3
$$

for both matrices. If the reduced matrix $R$ has a complete set of eigenvectors, it obeys the condition (42) of the lemma, and (43), (60) give $\operatorname{dim} \operatorname{ker}(R)=3$ for systems I,II and $\operatorname{dim} \operatorname{ker}(R)=5$ for system III. Hence, the three eigenvectors (9-11) and the five eigenvectors (31-35) span the entire nullspaces of the corresponding matrices $R$.

Let (56) be a vector in the nullspace of the reduced matrix, that is, a linear combination of eigenvectors $(9-11)$ or (31-35). It is easy to verify that

$$
(u_{1}^{0}, \ldots, u_{N}^{0}, \underbrace{0, \ldots, 0}_{L})^{T}
$$


substituted into (13) or, correspondingly, (37) yields zero, so (61) is an eigenvector of the extended matrix associated with $c=0$. In addition to (61), the extended matrix has $L$ linearly independent eigenvectors associated with $c=0$ of the form

$$
(\underbrace{0, \ldots, 0}_{N}, \psi_{1}^{0}, \ldots, \psi_{L}^{0})^{T}
$$

Thus, it is shown that if the reduced matrix has $N$ linearly independent eigenvectors (56) associated with both $c \neq 0$ and $c=0$, then the extended matrix has $N+L$ linearly independent eigenvectors (57), (61), (62).

Now suppose that the extended matrix has a set of $N+L$ linearly independent eigenvectors. Without loss of generality, it may be assumed that this set contains $L$ vectors of the form (62) and $N$ vectors of the general form (57). Let (56) denote the first $N$ components of (57). Each $(N+L)$-component vector is a linear combination of the $N+L$ vectors (57), (62). Since the first $N$ components of the vectors (62) are zero, each $N$-component vector is a linear combination of the $N$ vectors (56). Therefore, these $N$ vectors form a basis for $\mathbb{R}^{N}$ and are linearly independent. At the same time, they are eigenvectors of the reduced matrix: it is easy to check that any nonzero part (56) of the eigenvector (57) of the extended matrix is an eigenvector of the reduced matrix associated with the same $c$.

\section{Hyperbolicity and the acoustic matrix}

The implication of Proposition 1 is that information about hyperbolicity of the reduced system is sufficient to draw a conclusion about hyperbolicity of the extended system. The verification of hyperbolicity can be simplified further using the fact that the reduced matrix has the block structure (8) and a known nullspace.

For a nonzero eigenvalue of the reduced matrix of system I, substituting $\sigma_{j i}^{0}$ from (6) into (5) shows that the components $v_{i}^{0}$ satisfy the system

$$
\frac{1}{\varrho} A_{i k} v_{k}^{0}=c^{2} v_{i}^{0}, \quad i=1,2,3,
$$

where

$$
A_{i k}=C_{j i k l} n_{j} n_{l}
$$

are the components of the acoustic tensor. To ensure that (63) constitutes an eigenvalue problem and $c^{2}$ is therefore an eigenvalue of the matrix $A_{i k} / \varrho$, we need to show that the components $v_{i}^{0}$ in the eigenvector of the original matrix are not all zero. To see that this is indeed the case, substitute $v_{i}^{0}=0$ into (6) to obtain $\sigma_{j i}^{0}=0$. The equalities $v_{i}^{0}=0$ and $\sigma_{j i}^{0}=0$ together are impossible because $v_{i}^{0}, \sigma_{j i}^{0}$ are the components of an eigenvector.

Similarly to system I, substituting $\sigma_{j i}^{0}$ from (21) into (5) for system II with $c \neq 0$ yields the eigenvalue problem (63) with the acoustic tensor

$$
A_{i k}=\left(C_{j i k l}+\frac{K_{f}}{n} \delta_{j i} \delta_{k l}\right) n_{j} n_{l}
$$

For system III with $c \neq 0$, we write, according to (27), $v_{f i}^{0}=w_{1}^{0} n_{i}$ with a new single component $w_{1}^{0}$ instead of $v_{f 1}^{0}, v_{f 2}^{0}, v_{f 3}^{0}$, and eliminate $\sigma_{j i}^{0}, p_{f}^{0}$ from (26-29), which yields an eigenvalue problem for the components $v_{s 1}^{0}, v_{s 2}^{0}, v_{s 3}^{0}, w_{1}^{0}$ :

$$
\begin{aligned}
& {\left[\frac{1}{(1-n) \varrho_{s}} C_{j i k l} n_{j} n_{l}+\frac{K_{f}(1-n)}{n \varrho_{s}} n_{i} n_{k}\right] v_{s k}^{0}+\frac{K_{f}}{\varrho_{s}} n_{i} w_{1}^{0}=c^{2} v_{s i}^{0}, \quad i=1,2,3,} \\
& \frac{K_{f}(1-n)}{n \varrho_{f}} n_{k} v_{s k}^{0}+\frac{K_{f}}{\varrho_{f}} w_{1}^{0}=c^{2} w_{1}^{0} .
\end{aligned}
$$

The $4 \times 4$ matrix of the eigenvalue problem (66), (67) will be referred to as the acoustic matrix. For convenience, the matrix with the components $A_{i k} / \varrho$ for systems I,II will also be called the acoustic matrix.

Equations (63-67) lead to the well-known conclusion that the squared characteristic speeds are the eigenvalues of the acoustic matrix. Notice that, strictly speaking, this statement concerns only nonzero characteristic 
speeds, because there always exists a zero characteristic speed which need not be an eigenvalue of the acoustic matrix. The converse formulation is correct without restrictions: if $c^{2}$ is an eigenvalue of the acoustic matrix, then $\pm c$ are the characteristic speeds. Consequently, the dynamic equations lose hyperbolicity if an eigenvalue of the acoustic matrix becomes negative or complex.

The acoustic matrix allows us to find the characteristic speeds but does not immediately reveal whether the original matrix of the system is diagonalizable or not. In particular, it still remains unclear what happens if the acoustic matrix has a multiple positive eigenvalue or if one eigenvalue becomes zero while the others are real and positive. The answers are given by the following proposition which establishes a connection between the acoustic matrix and the diagonalizability of the original matrix. The proposition is valid for the reduced systems I-III of Table 1 with the acoustic tensors (64), (65) and the acoustic matrix of (66), (67).

Proposition 2 A reduced system is hyperbolic if and only if for each $\mathbf{n}$ the eigenvalues of the acoustic matrix are real and positive with a complete set of eigenvectors.

Proof First consider in more detail the transition from the reduced matrix of system III to the acoustic matrix determined by (66), (67). For a given $\mathbf{n}$, introduce new components $w_{1}^{0}, w_{2}^{0}, w_{3}^{0}$ instead of $v_{f 1}^{0}, v_{f 2}^{0}, v_{f 3}^{0}$ with the relations

$$
\left(\begin{array}{l}
w_{1}^{0} \\
w_{2}^{0} \\
w_{3}^{0}
\end{array}\right)=\left(\begin{array}{lll}
n_{1} & n_{2} & n_{3} \\
s_{1} & s_{2} & s_{3} \\
q_{1} & q_{2} & q_{3}
\end{array}\right)\left(\begin{array}{c}
v_{f 1}^{0} \\
v_{f 2}^{0} \\
v_{f 3}^{0}
\end{array}\right), \quad\left(\begin{array}{c}
v_{f 1}^{0} \\
v_{f 2}^{0} \\
v_{f 3}^{0}
\end{array}\right)=\left(\begin{array}{lll}
n_{1} & s_{1} & q_{1} \\
n_{2} & s_{2} & q_{2} \\
n_{3} & s_{3} & q_{3}
\end{array}\right)\left(\begin{array}{c}
w_{1}^{0} \\
w_{2}^{0} \\
w_{3}^{0}
\end{array}\right),
$$

where $s_{i}, q_{i}$ are the components of the unit vectors $\mathbf{s}, \mathbf{q}$ orthogonal to each other and to the vector $\mathbf{n}$. Three equations (27), which contain $v_{f i}^{0}$, yield three equations with $w_{i}^{0}$ :

$$
\frac{1}{\varrho_{f}} p_{f}^{0}=c w_{1}^{0}, \quad 0=c w_{2}^{0}, \quad 0=c w_{3}^{0} .
$$

Equation (29) becomes

$$
K_{f}\left(\frac{1-n}{n}\right) n_{k} v_{s k}^{0}+K_{f} w_{1}^{0}=c p_{f}^{0} .
$$

Divide the vector components into two groups, but differently from (30):

$$
(\underbrace{v_{s 1}^{0}, v_{s 2}^{0}, v_{s 3}^{0}, w_{1}^{0}}_{N_{1}}, \underbrace{w_{2}^{0}, w_{3}^{0}, \sigma_{11}^{0}, \sigma_{12}^{0}, \sigma_{13}^{0}, \sigma_{22}^{0}, \sigma_{33}^{0}, \sigma_{23}^{0}, p_{f}^{0}}_{N_{2}})^{T} .
$$

The matrix $R$ of the new system (26), (28), (69), (70) has the structure (8) and is connected with the old matrix by a similarity transformation. Since similarity preserves diagonalizability, the new matrix $R$ will be considered hereafter instead of the original reduced matrix of system III. The new matrix $R$ has 5 linearly independent eigenvectors associated with $c=0$ similar to (31-35):

$$
\begin{array}{llll}
v_{s i}^{0}=0, & w_{i}^{0}=0, \quad \sigma_{j i}^{0}=s_{j} s_{i}, \quad p_{f}^{0}=0, \\
v_{s i}^{0}=0, & w_{i}^{0}=0, \quad \sigma_{j i}^{0}=q_{j} q_{i}, \quad p_{f}^{0}=0, \\
v_{s i}^{0}=0, & w_{i}^{0}=0, \quad \sigma_{j i}^{0}=s_{j} q_{i}+s_{i} q_{j}, \quad p_{f}^{0}=0, \\
v_{s i}^{0}=0, & w_{1}^{0}=0, \quad w_{2}^{0}=1, \quad w_{3}^{0}=0, \quad \sigma_{j i}^{0}=0, \quad p_{f}^{0}=0, \\
v_{s i}^{0}=0, & w_{1}^{0}=0, \quad w_{2}^{0}=0, \quad w_{3}^{0}=1, \quad \sigma_{j i}^{0}=0, \quad p_{f}^{0}=0 .
\end{array}
$$

The submatrix $Y$ of the new matrix $R$ is

$$
Y_{4 \times 9}=-\frac{1}{(1-n) \varrho_{s}}\left(\begin{array}{ccccccccc}
0 & 0 & n_{1} & n_{2} & n_{3} & 0 & 0 & 0 & -(1-n) n_{1} \\
0 & 0 & 0 & n_{1} & 0 & n_{2} & 0 & n_{3} & -(1-n) n_{2} \\
0 & 0 & 0 & 0 & n_{1} & 0 & n_{3} & n_{2} & -(1-n) n_{3} \\
0 & 0 & 0 & 0 & 0 & 0 & 0 & 0 & -(1-n) \varrho_{s} / \varrho_{f}
\end{array}\right) .
$$

The rank of the matrix (77) is 4 for any $\mathbf{n}$. 
It was shown in the proof of Proposition 1 of Sect. 3 that if the reduced matrix $R$ of system I or II has a complete set of eigenvectors, then $\operatorname{dim} \operatorname{ker}(R)=3$, and the three eigenvectors (9-11) span the entire nullspace of $R$. For the new matrix $R$ of system III, the same argument gives $\operatorname{dim} \operatorname{ker}(R)=5$, so the five eigenvectors (72-76) also span the entire nullspace of $R$. $R$ :

Let $a=\left(a_{1}, \ldots, a_{N_{1}}\right)^{T}, b=\left(b_{1}, \ldots, b_{N_{2}}\right)^{T}$ denote two vectors which form an eigenvector $u$ of the matrix

$$
u=\left(a_{1}, \ldots, a_{N_{1}}, b_{1}, \ldots, b_{N_{2}}\right)^{T},
$$

where $N_{1}=3, N_{2}=6$ for systems I,II and $N_{1}=4, N_{2}=9$ for system III. The eigenvalue problem $R u=c u$ can be written as two systems:

$$
X a=c b, \quad Y b=c a .
$$

Suppose that the matrix $R$ has $N$ real linearly independent eigenvectors $u$ with subvectors $a, b$. Then each $N_{1}$-component vector is a linear combination of the vectors $a$. The eigenvectors (9-11) and (72-76), which span the nullspaces, reveal that the vectors $a$ associated with $c=0$ are zero, so each $N_{1}$-component vector is actually a linear combination of the vectors $a$ associated with nonzero eigenvalues. This set of vectors $a$ must therefore contain a basis for $\mathbb{R}^{N_{1}}$. At the same time, equations (79) show that each vector $a$ satisfies the system $Y X a=c^{2} a$. It is easy to verify that the $N_{1} \times N_{1}$ matrix $Y X$ is the acoustic matrix. Hence, the acoustic matrix has a complete set of eigenvectors associated with real positive eigenvalues.

Now suppose that the eigenvalues $c^{2}$ of the acoustic matrix are real and positive and there exists a complete set of eigenvectors $a$. As follows from (79), setting $b=X a /( \pm c)$ for each pair $c^{2}, a$ makes (78) an eigenvector of $R$ associated with $\pm c$. If the $N_{1}$ eigenvalues $c^{2}$ are different, then the $2 N_{1}$ eigenvectors $u$ are linearly independent because they are associated with the different eigenvalues $\pm c$. If there is a multiple eigenvalue $c^{2}$ of the acoustic matrix, we should take linearly independent eigenvectors $a$ associated with that $c^{2}$ to have linearly independent eigenvectors $u$. In any case, there exist $2 N_{1}$ linearly independent eigenvectors $u$ of $R$ associated with $c \neq 0$. As we already know, the matrix $R$ has $N-2 N_{1}$ linearly independent eigenvectors associated with $c=0$ ( 3 for systems I,II and 5 for system III), which makes $N$ linearly independent eigenvectors altogether.

\section{Concluding remarks}

The two propositions of the paper applied successively reduce the verification of hyperbolicity for a plastic solid to the solution of the eigenvalue problem for the corresponding acoustic matrix. This result is not only of theoretical interest but is also useful for numerical applications, as the acoustic matrix is smaller than the whole matrix of the system. What is needed is only to check that the plasticity model conforms with the assumptions (i-iii) of Sect. 2.2 and with equations (12), (36).

As follows from Proposition 2, if the eigenvalues of the acoustic matrix are positive and different, then the equations are hyperbolic. A multiple positive eigenvalue per se does not cause a loss of hyperbolicity if there still exists a complete set of eigenvectors. Another corollary of Proposition 2 is that the equations cannot be hyperbolic if the acoustic matrix is singular. In this case, as seen from the proof, the matrix of the system does not have a complete set of eigenvectors (it is defective).

A further problem of interest is to investigate the relation between hyperbolicity of the equations for a dry porous solid and hyperbolicity for the same but fluid-saturated solid. Since the equations for zero and nonzero permeability for a saturated solid are different, there are three systems of equations to be compared. According to the effective stress principle, the constitutive description of the solid in all three cases involves the same stiffness tensor of the skeleton, $C_{j i k l}$. Nevertheless, the three systems in general do not agree with each other from the viewpoint of hyperbolicity. It is shown in [9] that the equations for a saturated solid may lose hyperbolicity while the equations for the same but dry solid remain hyperbolic. Hence, the conditions of Proposition 2 for the acoustic tensor $A_{i k}$ of the dry solid to have real positive eigenvalues and a complete set of eigenvectors do not ensure hyperbolicity in the saturated case. Stronger conditions sufficient for hyperbolicity for the saturated solid are the symmetry and positive definiteness of the acoustic tensor of the skeleton. This issue is considered in [17].

Funding Open Access funding enabled and organized by Projekt DEAL. 
Open Access This article is licensed under a Creative Commons Attribution 4.0 International License, which permits use, sharing, adaptation, distribution and reproduction in any medium or format, as long as you give appropriate credit to the original author(s) and the source, provide a link to the Creative Commons licence, and indicate if changes were made. The images or other third party material in this article are included in the article's Creative Commons licence, unless indicated otherwise in a credit line to the material. If material is not included in the article's Creative Commons licence and your intended use is not permitted by statutory regulation or exceeds the permitted use, you will need to obtain permission directly from the copyright holder. To view a copy of this licence, visit http://creativecommons.org/licenses/by/4.0/.

\section{Declarations}

Conflict of interest The author states that there is no conflict of interest.

\section{References}

1. Hill, R.: Acceleration waves in solids. J. Mech. Phys. Solids 10, 1-16 (1962)

2. Rice, J.R.: The localization of plastic deformation. In: Koiter, W.T. (ed.) Theoretical and Applied Mechanics. Proc. 14th IUTAM Congress, pp. 207-220. North-Holland, Amsterdam (1976)

3. Loret, B., Prévost, J.H., Harireche, O.: Loss of hyperbolicity in elastic-plastic solids with deviatoric associativity. Eur. J. Mech. A/Solids 9(3), 225-231 (1990)

4. Loret, B., Harireche, O.: Acceleration waves, flutter instabilities and stationary discontinuities in inelastic porous media. J. Mech. Phys. Solids 39(5), 569-606 (1991)

5. An, L., Schaeffer, D.G.: The flutter instability in granular flow. J. Mech. Phys. Solids 40(3), 683-698 (1992)

6. Bigoni, D., Zaccaria, D.: On the eigenvalues of the acoustic tensor in elastoplasticity. Eur. J. Mech. A/Solids 13(5), 621-638 (1994)

7. Osinov, V.A.: Theoretical investigation of large-amplitude waves in granular soils. Soil Dyn. Earthq. Eng. 17(1), 13-28 (1998)

8. Osinov, V.A., Gudehus, G.: Dynamics of hypoplastic materials: theory and numerical implementation. In: Hutter, K., Kirchner, N. (eds.) Dynamic Response of Granular and Porous Materials under Large and Catastrophic Deformations, pp. $265-284$. Springer, Berlin (2003)

9. Osinov, V.A.: On well-posedness of the dynamic problem for an anisotropic fluid-saturated solid. Arch. Appl. Mech. 79, 69-80 (2009)

10. Evans, L.C.: Partial Differential Equations, 2nd edn. American Mathematical Society, Providence (2010)

11. Zauderer, E.: Partial Differential Equations of Applied Mathematics, 2nd edn. Wiley, New York (1998)

12. Simo, J.C., Hughes, T.J.R.: Computational Inelasticity. Springer, New York (1998)

13. Zienkiewicz, O.C., Chan, A.H.C., Pastor, M., Schrefler, B.A., Shiomi, T.: Computational Geomechanics with Special Reference to Earthquake Engineering. Wiley, Chichester (1999)

14. Osinov, V.A.: Blast-induced waves in soil around a tunnel. Arch. Appl. Mech. 81, 543-559 (2011)

15. Horn, R.A., Johnson, C.R.: Matrix Analysis. Cambridge University Press, Cambridge (1985)

16. Meyer, C.D.: Matrix Analysis and Applied Linear Algebra. SIAM, Philadelphia (2000)

17. Osinov, V.A.: Sufficient conditions for hyperbolicity and consistency of the dynamic equations for fluid-saturated solids. Arch. Appl. Mech. (2021). https://doi.org/10.1007/s00419-021-01904-6

Publisher's Note Springer Nature remains neutral with regard to jurisdictional claims in published maps and institutional affiliations. 